\title{
POSTERS
}

Geophysical Research Abstracts Vol. 14, EGU2012-2365, 2012 EGU General Assembly 2012 C Author(s) 2012

\section{PLASMA DIFFUSION IN SELF-CONSISTENT FLUCTUATIONS}

\section{R. SMETS (1), G. BELMONT (1), N. AUNAI $(1,2)$}

(1) LPP, Ecole Polytechnique/CNRS, Space Physics, Palaiseau, France(gerard.belmont@lpp.polytechnique.fr), (2) Space Weather Laboratory, Code 674, NASAGoddard Space Flight Center, Greenbelt, Maryland 20771, USA

The problem of particle diffusion in position space, as a consequence of electromagnetic fluctuations is addressed. Numerical results obtained with a self-consistent hybrid code are presented, and a method to calculate diffusion coefficient in the direction perpendicular to the mean magnetic field is proposed. The diffusion is estimated for two different types of fluctuations. The first type (resulting from an agyrotropic initial setting)is stationary, wide band white noise, and associated to Gaussian probability distribution function for the magnetic fluctuations. The second type (resulting from a Kelvin-Helmholtz instability) is non-stationary, with a power-law spectrum, and a non-Gaussian probability distribution function. The results of the study allow revisiting the question of loading particles of solar wind origin in the Earth magnetosphere . 